\title{
Shape from bandwidth
}

The 2-D orthogonal projection case

Adam Scholefield, Benjamín Béjar Haro and Martin Vetterli 
Bandwidth

LCAV

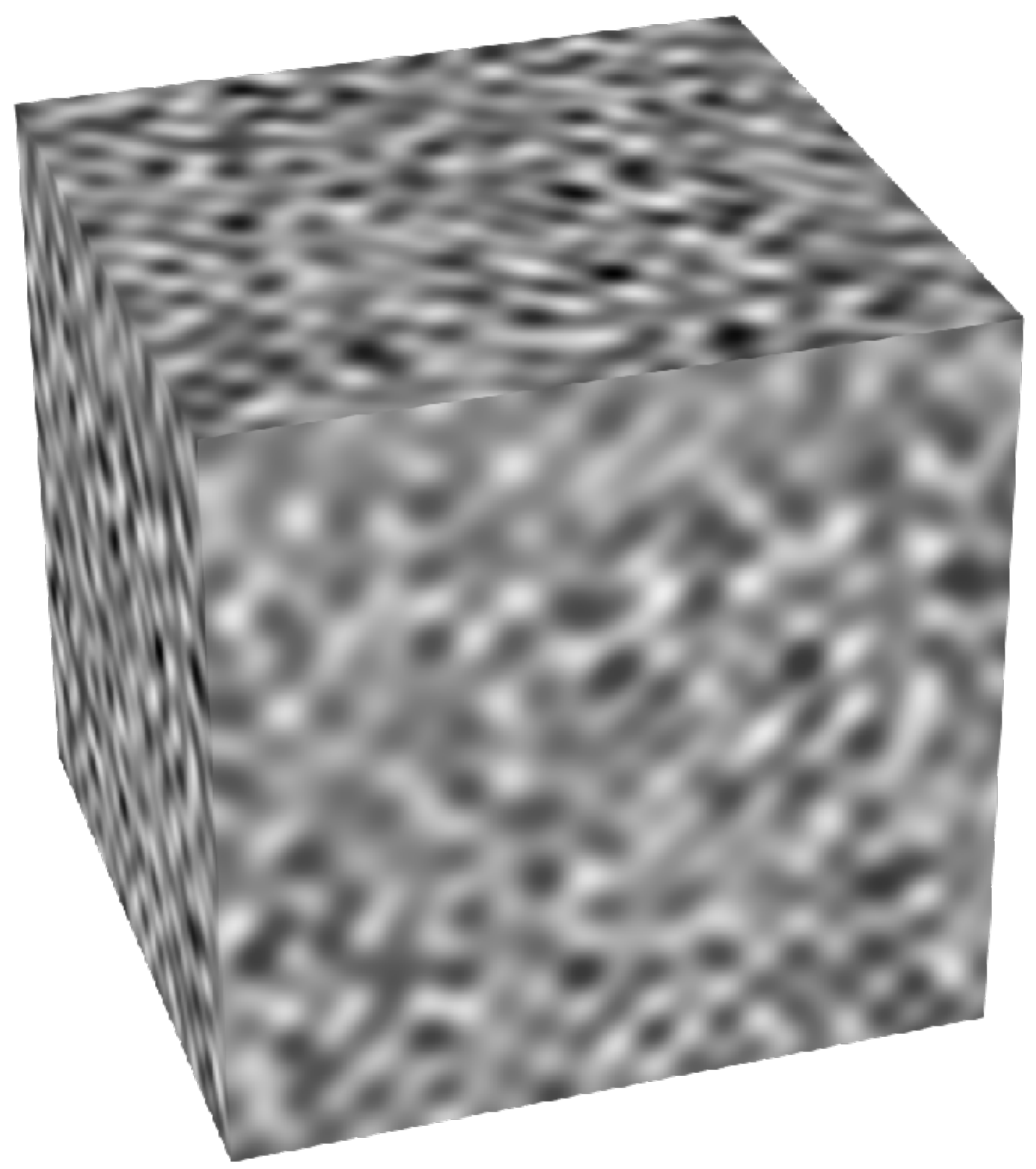


2-D Orthogonal projection case

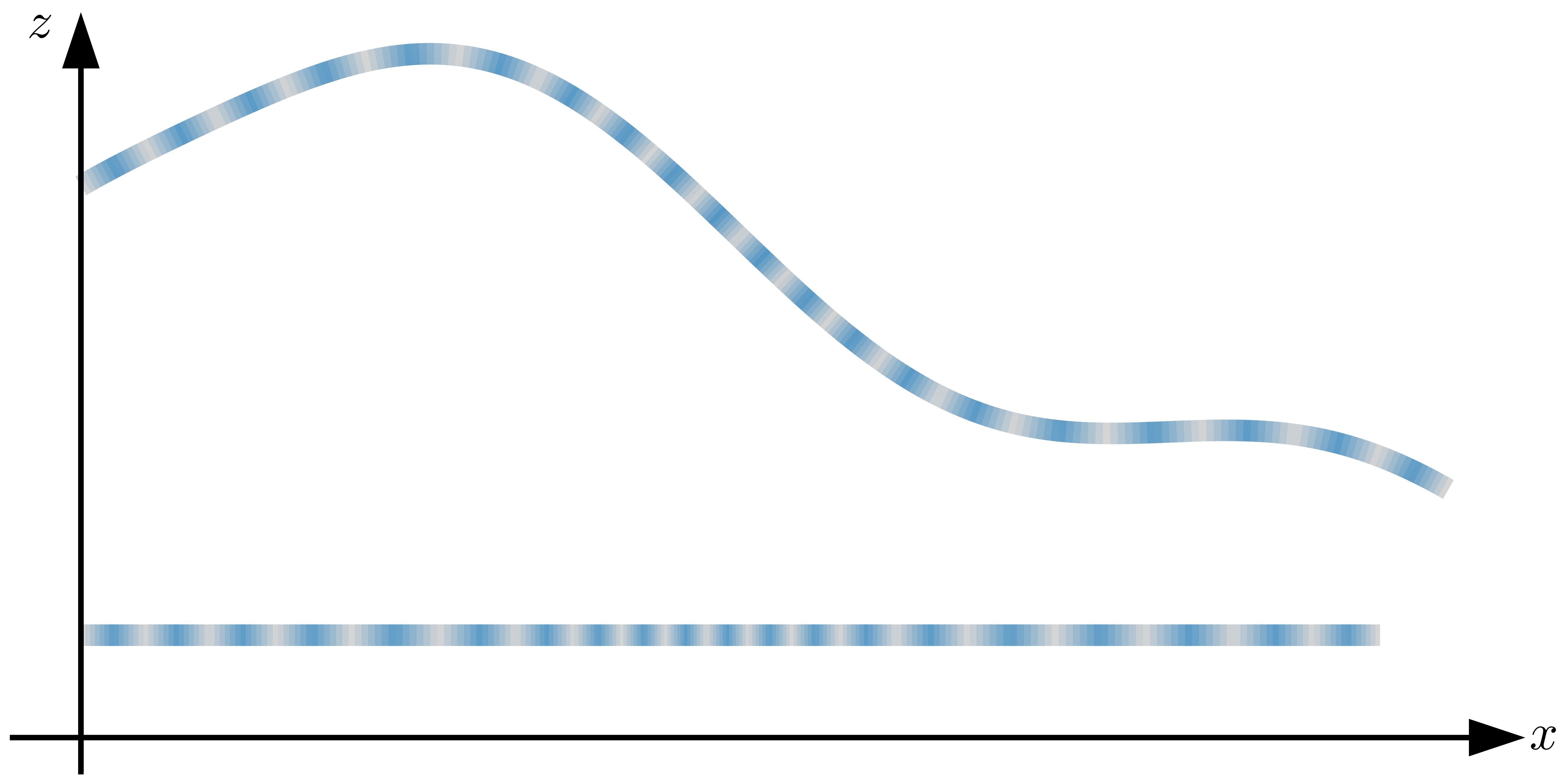


- Problem setup

- Algorithm overview:

- Observation to arc-length

- Arc-length to surface (ambiguities)

- Uniqueness result

- "Practical" experiment

- Conclusions and future work 


\section{Setup}




\section{Setup}

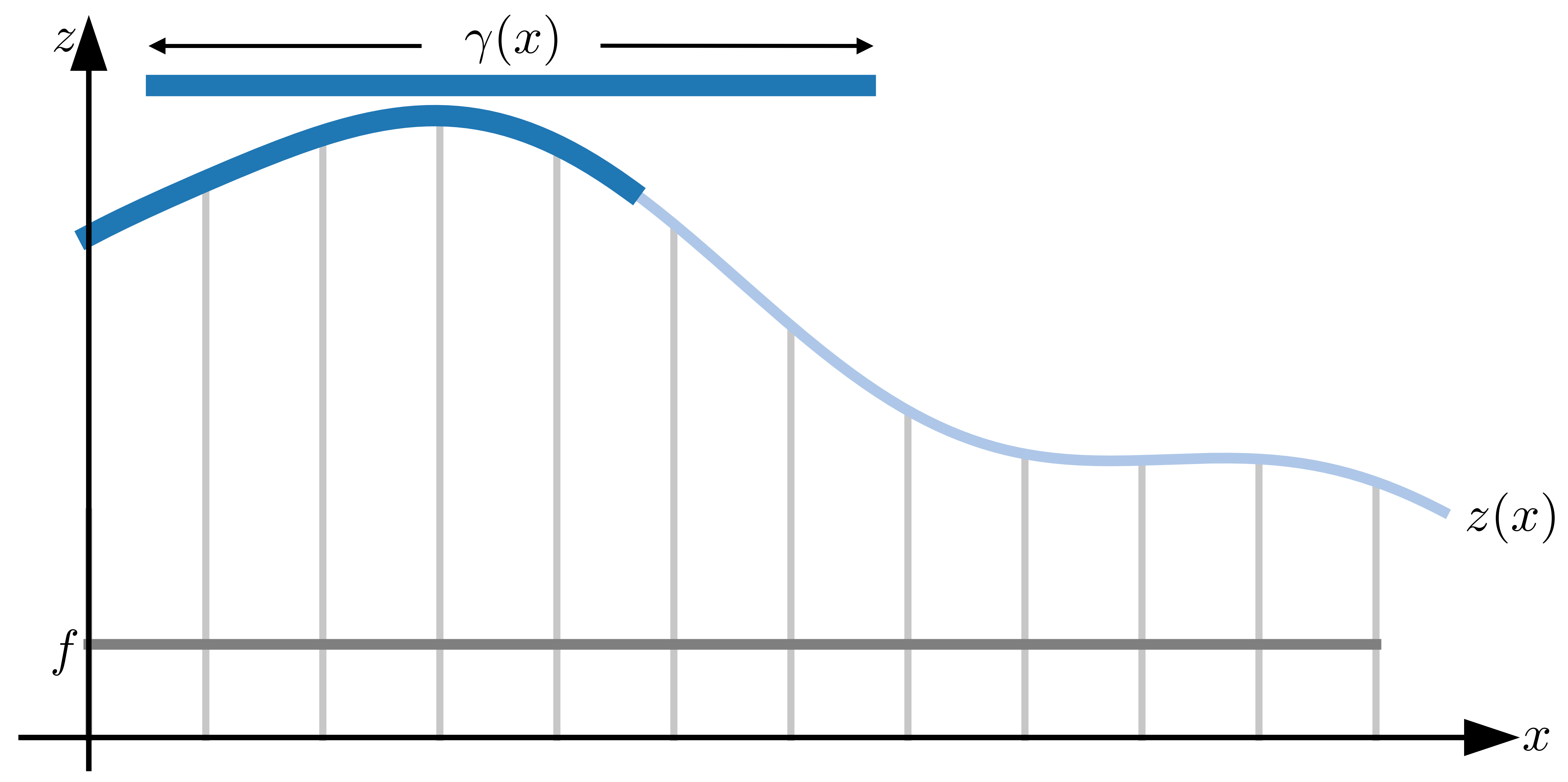


Setup

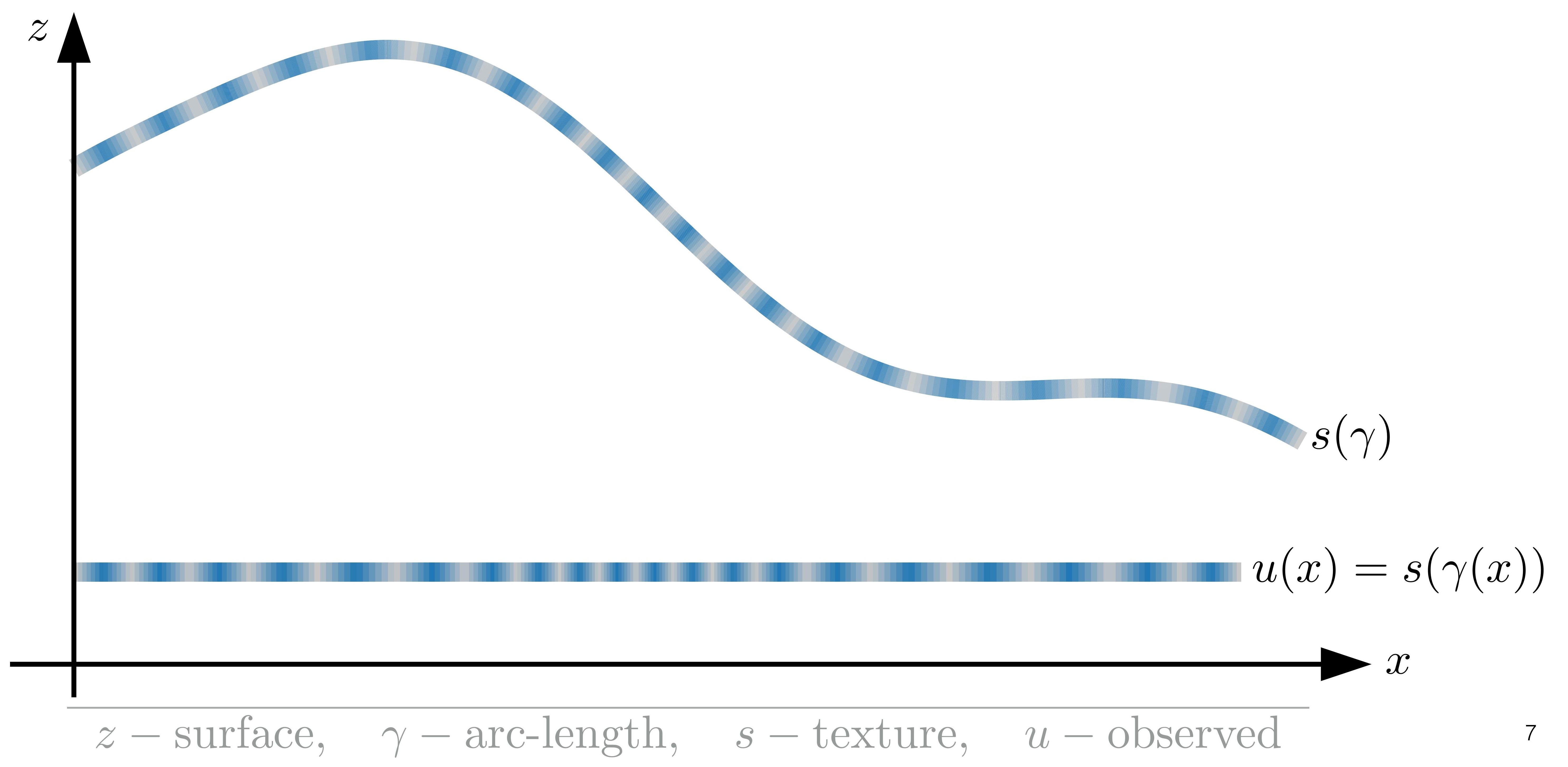


Algorithm overview 


\section{Observation to arc-length}

$$
\begin{gathered}
u(x)=s(\gamma(x)) \\
u\left(x ; x_{0}\right):=s\left(\gamma\left(x_{0}\right)+\gamma^{\prime}\left(x_{0}\right)\left(x-x_{0}\right)\right) \\
\mathcal{F}\left[u\left(x ; x_{0}\right)\right]=\frac{1}{\gamma^{\prime}\left(x_{0}\right)} S\left(\frac{\omega}{\gamma^{\prime}\left(x_{0}\right)}\right) e^{j \omega\left(\gamma\left(x_{0}\right) / \gamma^{\prime}\left(x_{0}\right)-x_{0}\right)}
\end{gathered}
$$

$s$ has bandwidth $B \Rightarrow u\left(x ; x_{0}\right)$ has bandwidth $\gamma^{\prime}\left(x_{0}\right) B$ 


\section{Observation to arc-length}

$$
\begin{gathered}
u(x)=s(\gamma(x)) \\
u\left(x ; x_{0}\right):=s\left(\gamma\left(x_{0}\right)+\gamma^{\prime}\left(x_{0}\right)\left(x-x_{0}\right)\right) \\
\mathcal{F}\left[u\left(x ; x_{0}\right)\right]=\frac{1}{\gamma^{\prime}\left(x_{0}\right)} S\left(\frac{\omega}{\gamma^{\prime}\left(x_{0}\right)}\right) e^{j \omega\left(\gamma\left(x_{0}\right) / \gamma^{\prime}\left(x_{0}\right)-x_{0}\right)}
\end{gathered}
$$

$s$ has bandwidth $B \Rightarrow u$ has local bandwidth $\gamma^{\prime}\left(x_{0}\right) B$ at $x_{0}$ 
Arc-length to surface gradient ${ }^{L C A V}$

$s$ has bandwidth $B \Rightarrow u$ has local bandwidth $\gamma^{\prime}\left(x_{0}\right) B$ at $x_{0}$

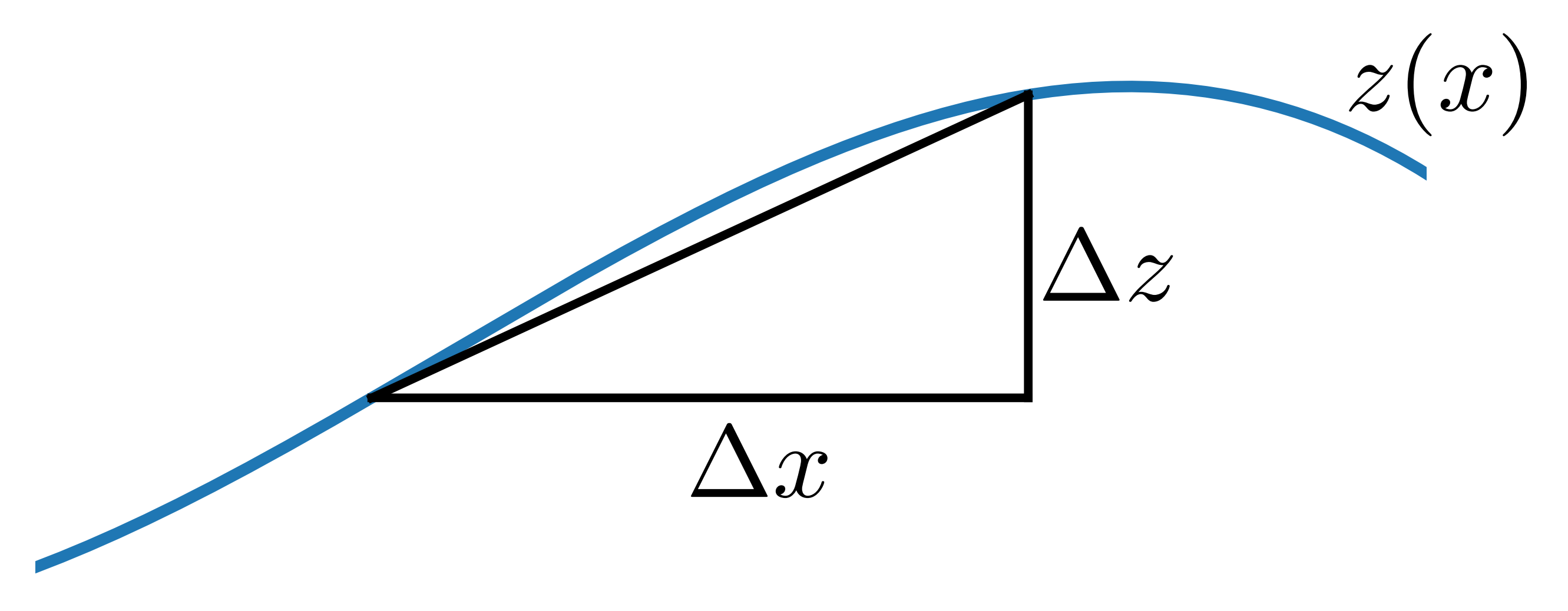

$$
(\Delta \gamma)^{2}=(\Delta x)^{2}+(\Delta z)^{2}
$$

$$
\left(\frac{d \gamma}{d x}\right)^{2}=1+\left(\frac{d z}{d x}\right)^{2} \Rightarrow\left|\frac{d z}{d x}\right|=\sqrt{\left(\frac{d \gamma}{d x}\right)^{2}-1}
$$




\section{Example}
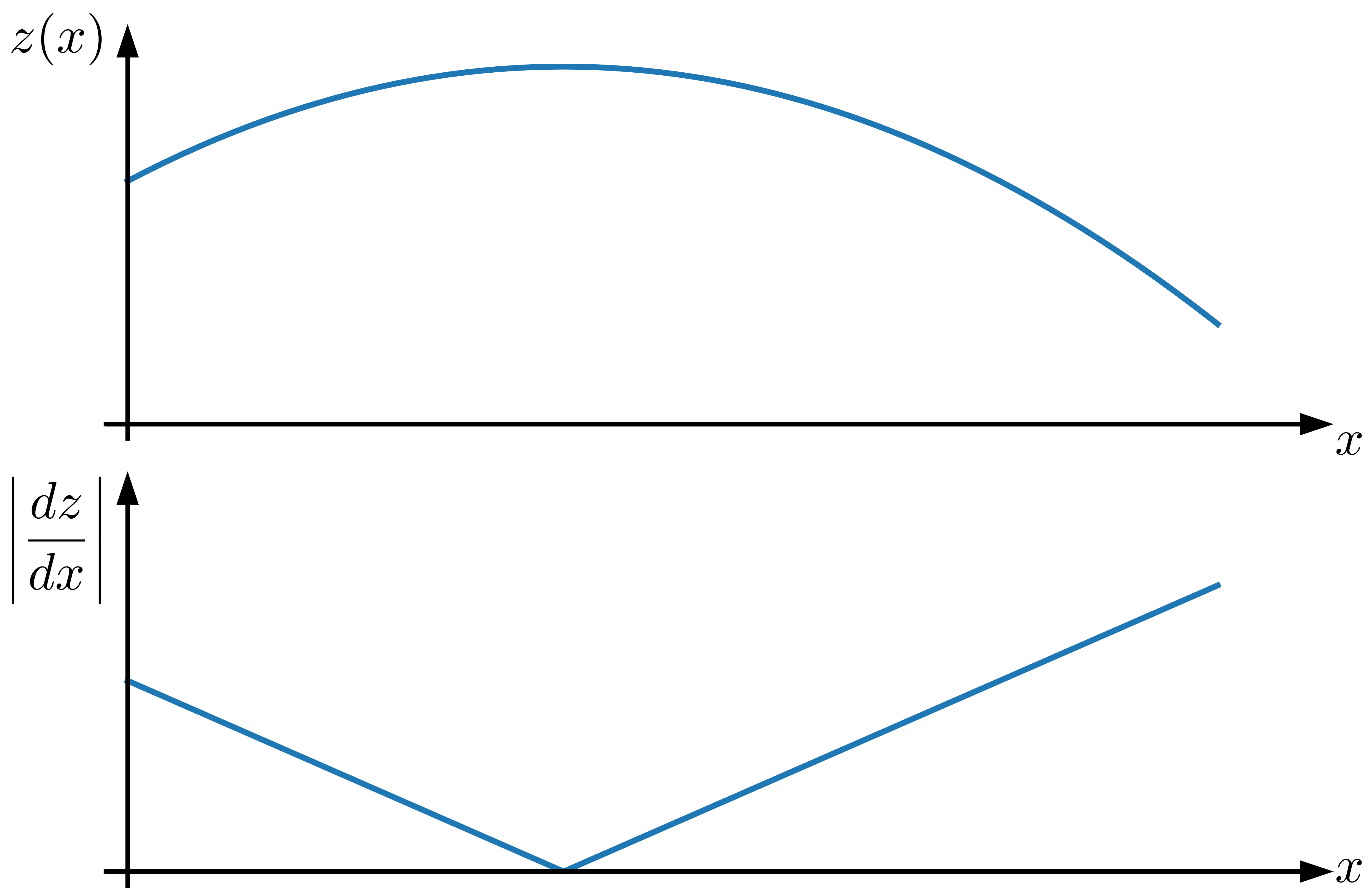


\section{Example}
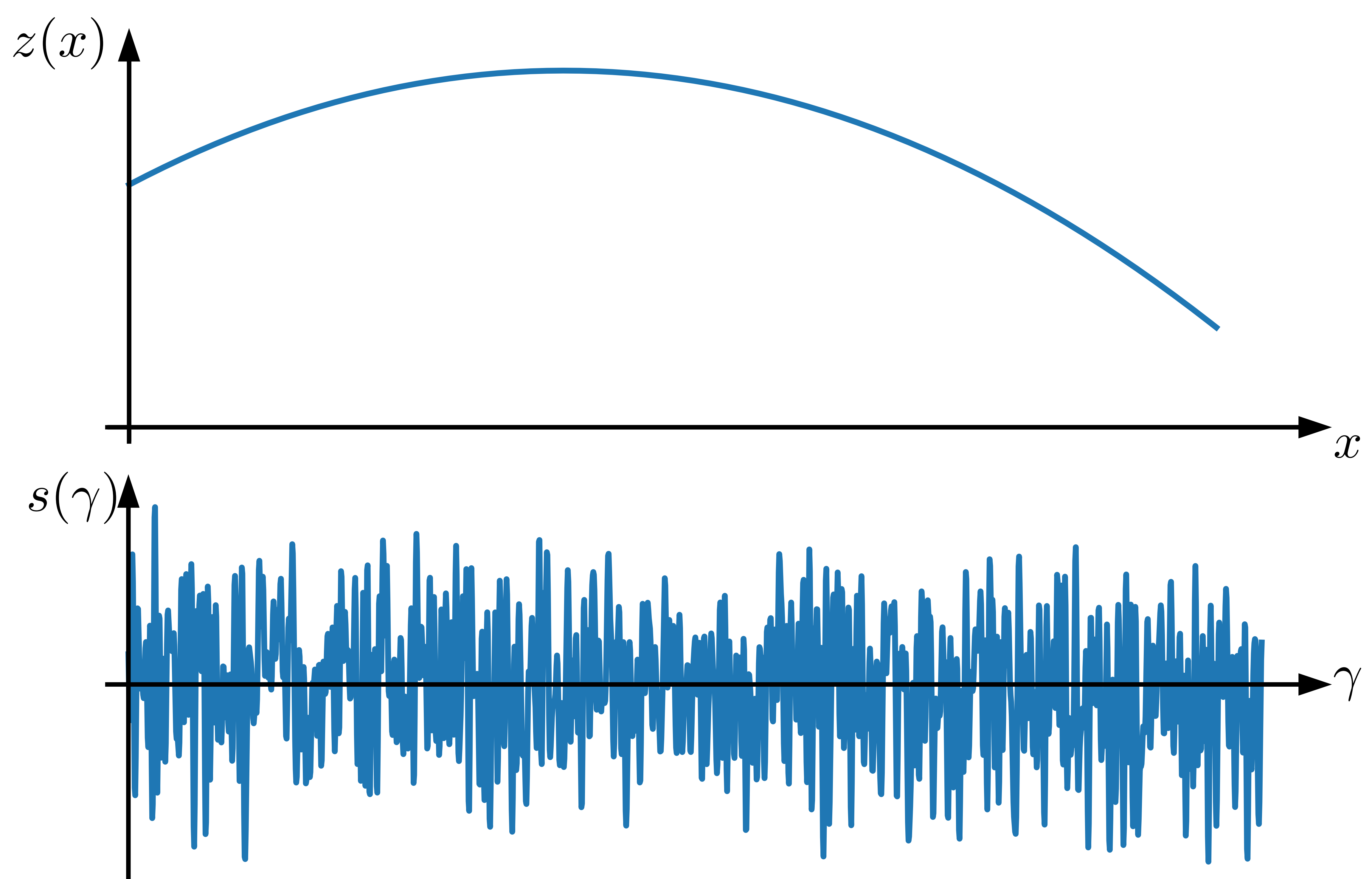


\section{Example}
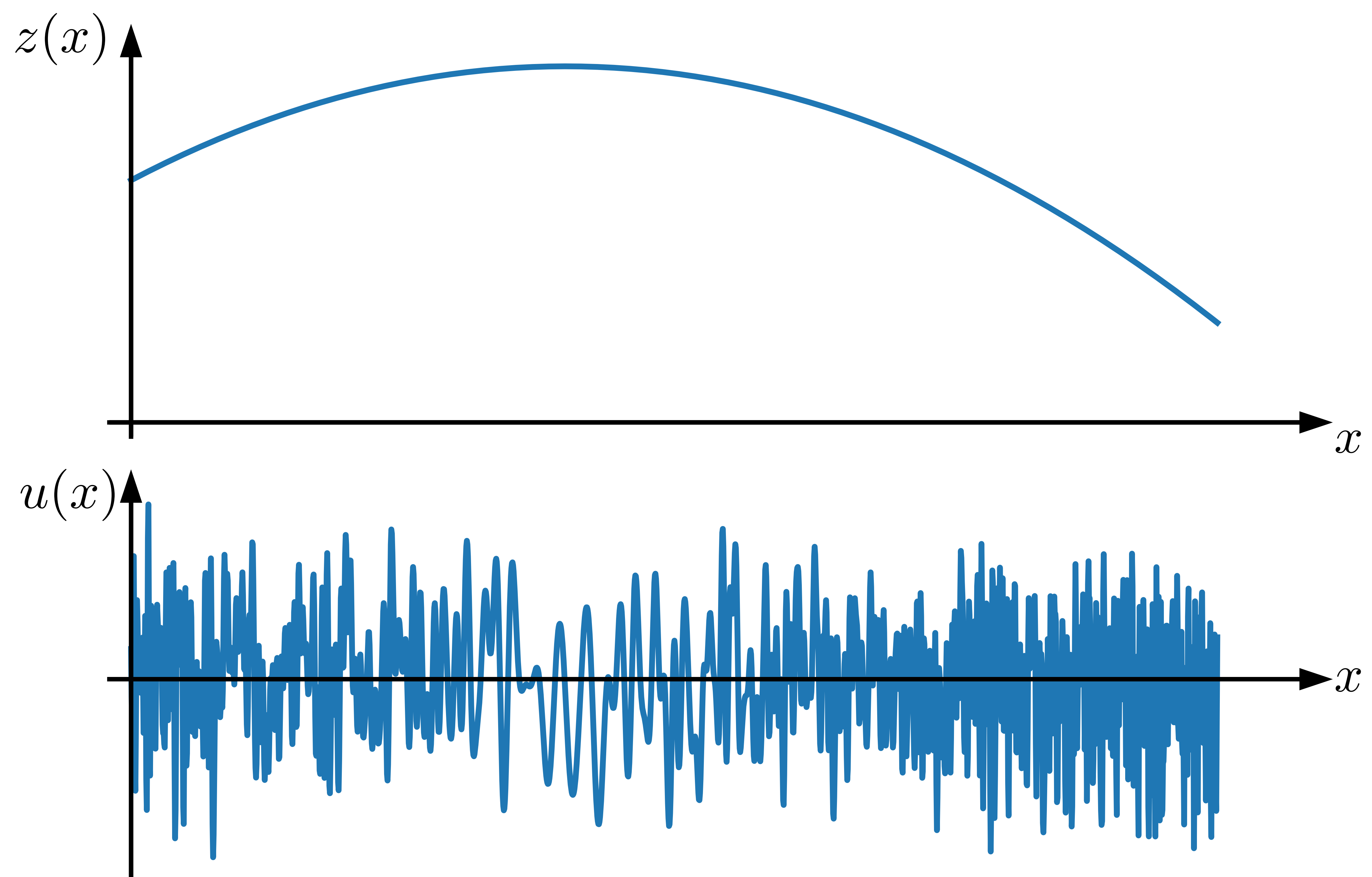
Example

LCAV

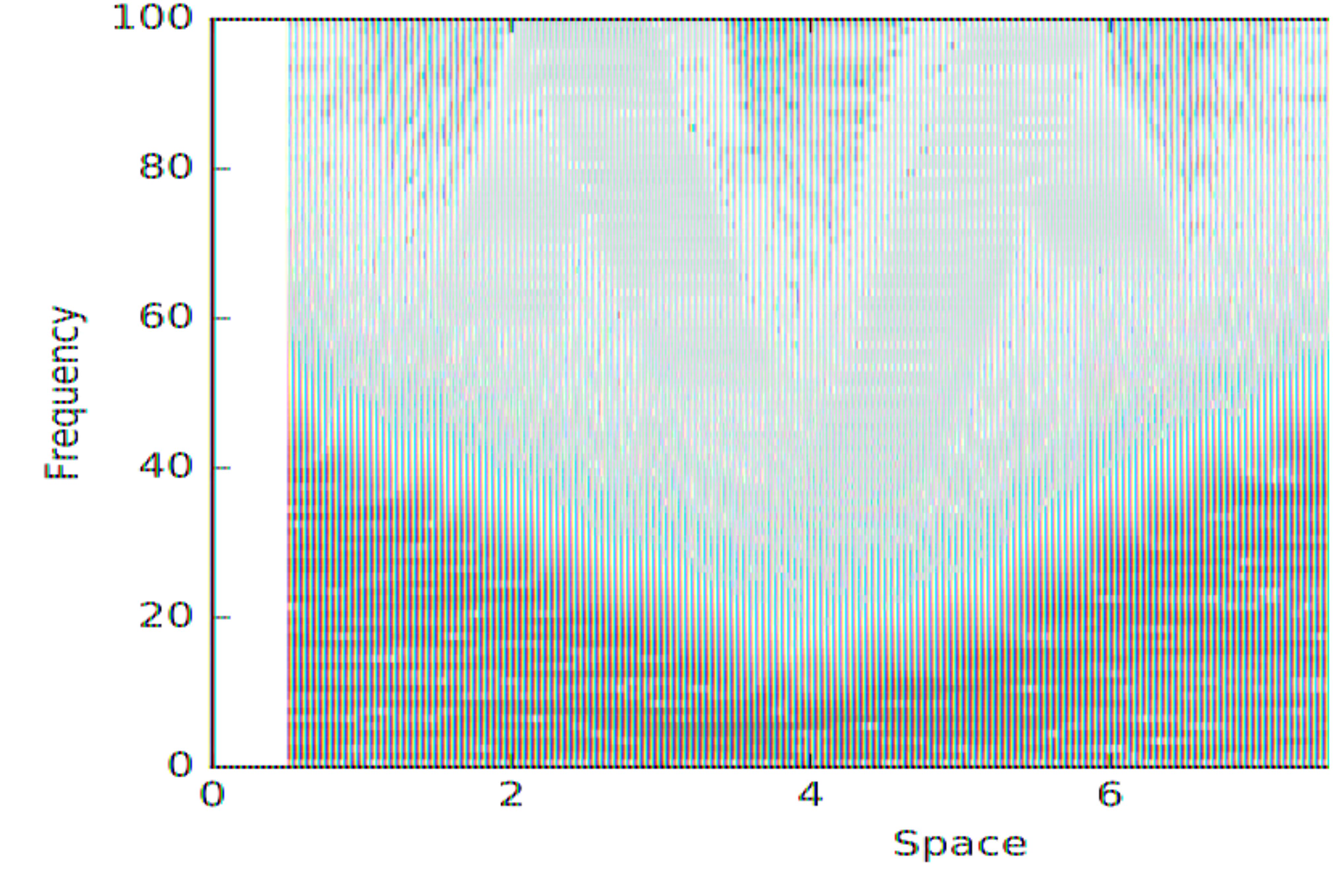




\section{Example}

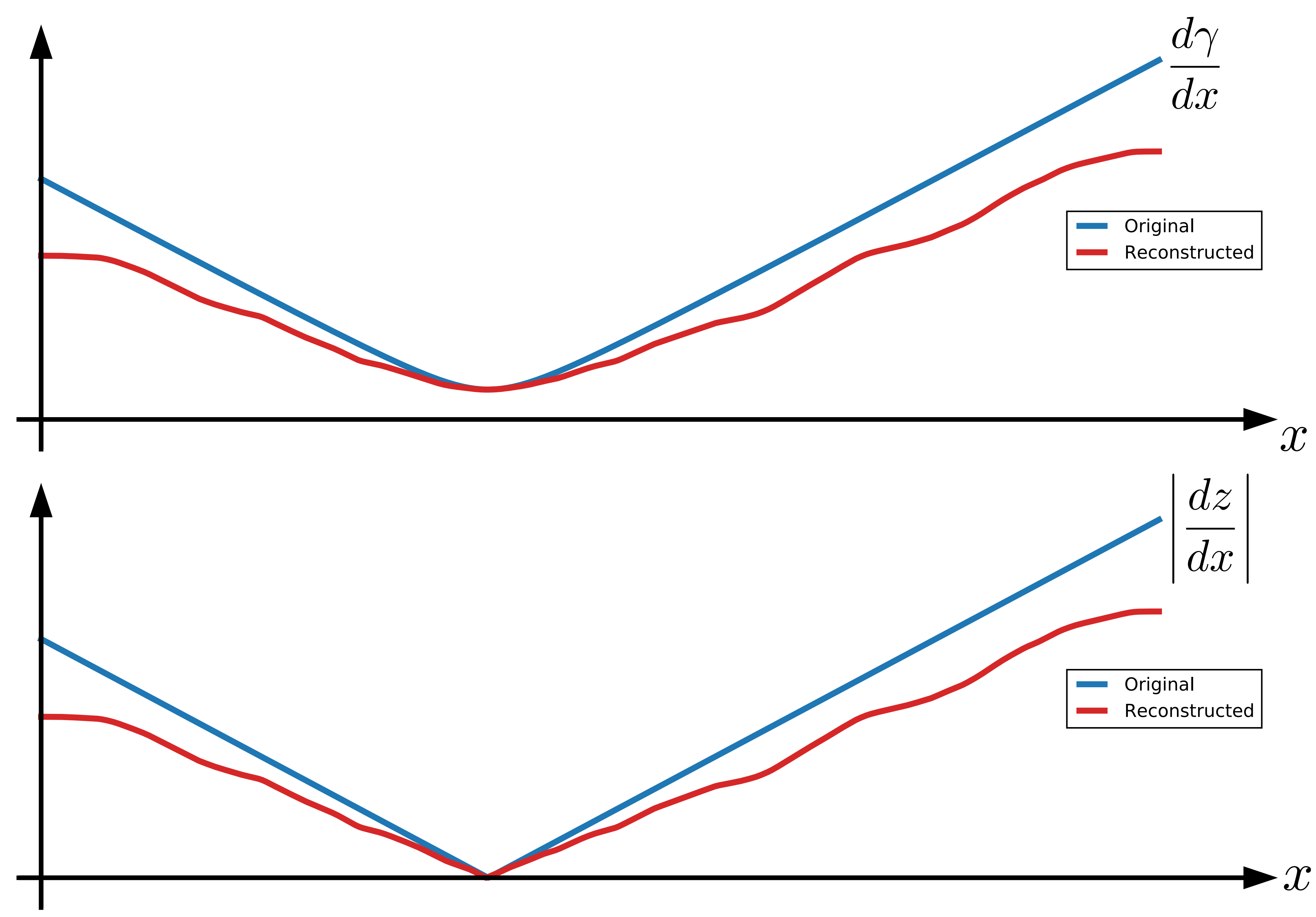




\section{Example}
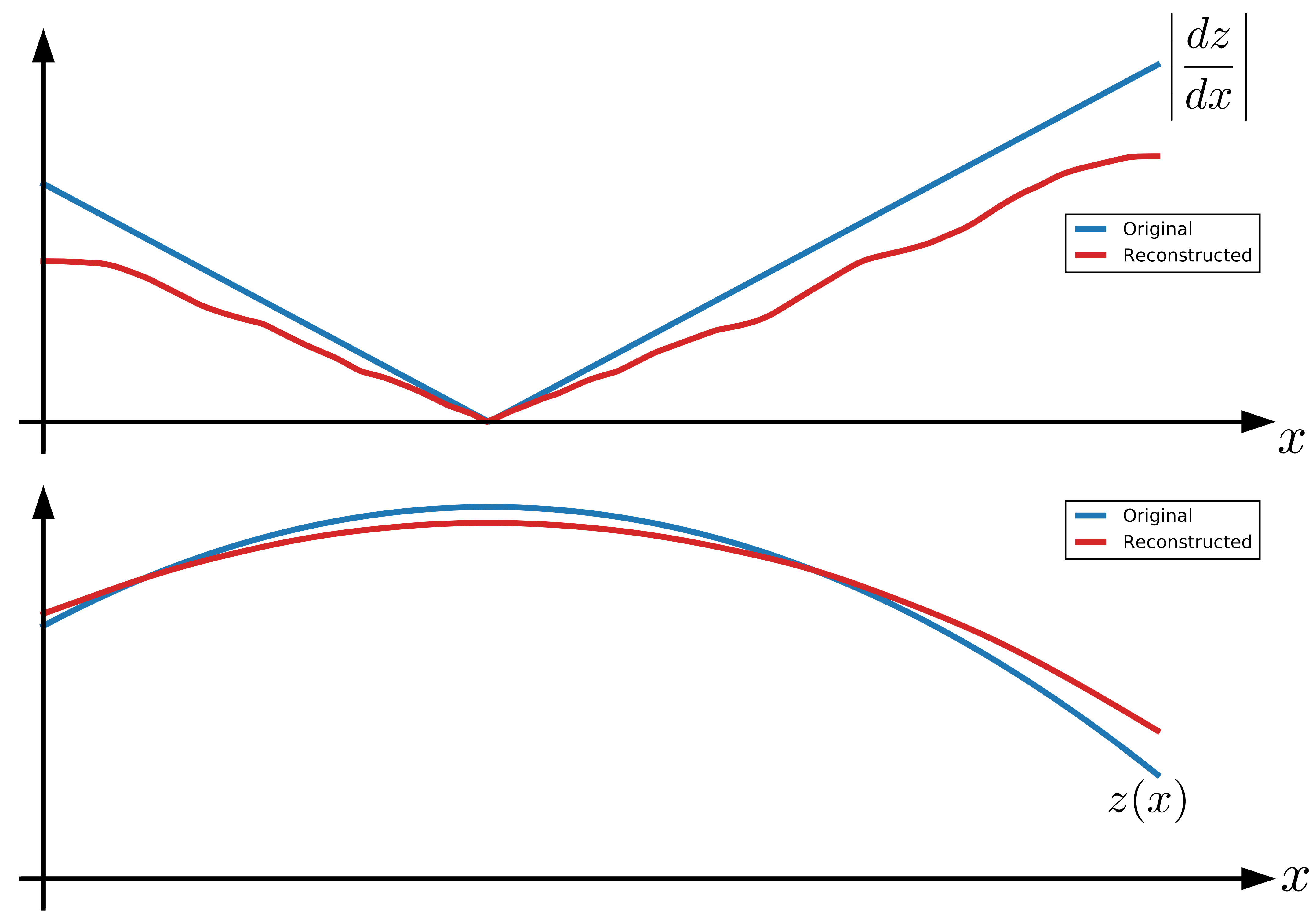


\section{Arc-length to surface (ambiguities)}




$$
\left(\frac{d \gamma}{d x}\right)^{2}=1+\left(\frac{d z}{d x}\right)^{2} \Rightarrow \hat{z}(x)=\int_{x_{\min }}^{x}\left|\frac{d z}{d x^{\prime}}\right| d x^{\prime}
$$
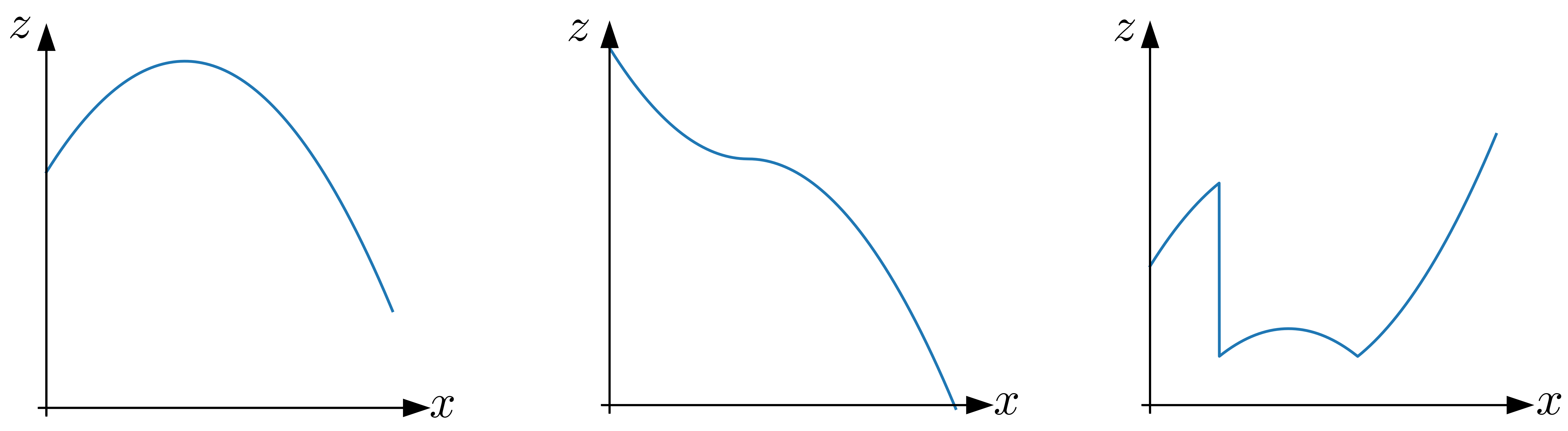

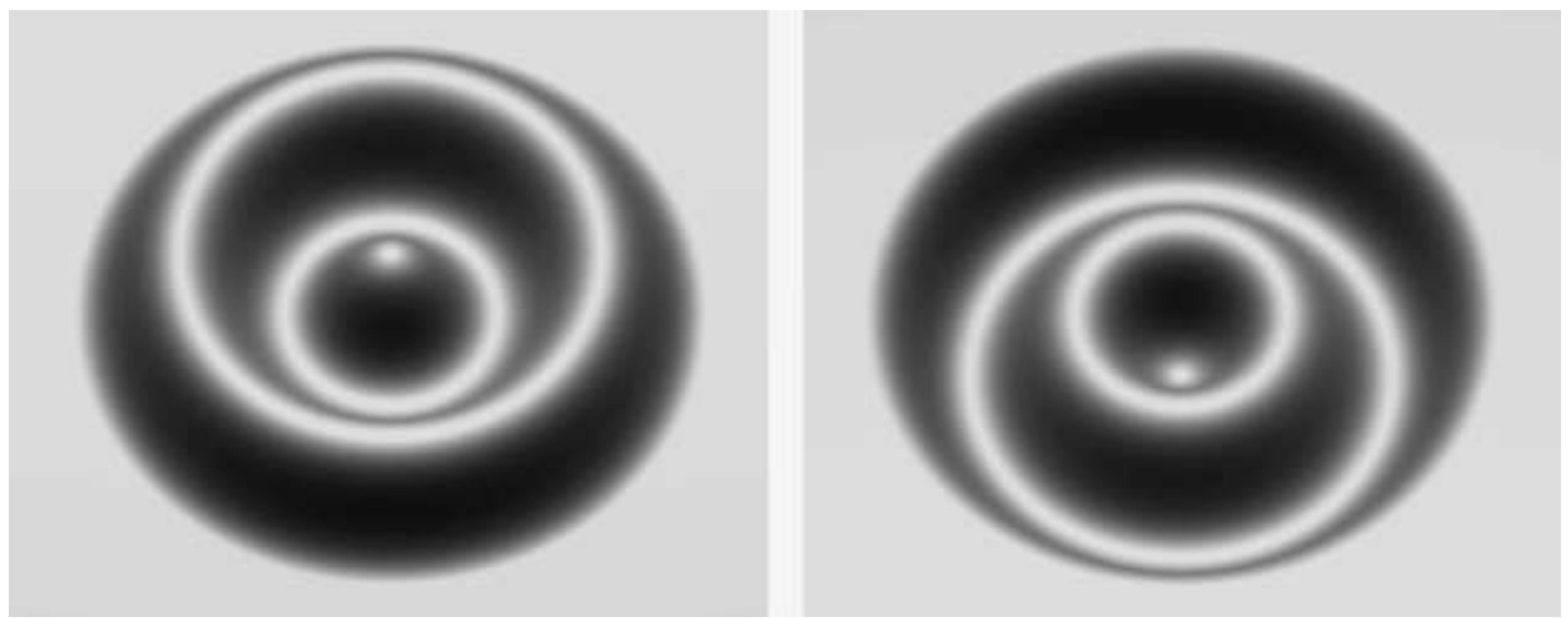
1. Compute spectrogram of observed signal

2. For each spatial value, estimate local BW

3. Divide local BW by minimum observed BW

4. Calculate $\left|\frac{d z}{d x}\right|=\sqrt{\left(\frac{d \gamma}{d x}\right)^{2}-1}$

5. Reconstruct all surfaces in the equivalence class 


\section{Uniqueness from Clark's conjecture}

Conjecture (Clark 1989). A warped bandlimited signal will be bandlimited if and only if the warping is affine.

Theorem (Xia and Zhang 1992). The conjecture is true if we restrict the warping to be entire on an interval.

Counter example (Azizi et. al.1999). Yves Meyer came up with a peculiar counter-example. 


\section{Experiment}




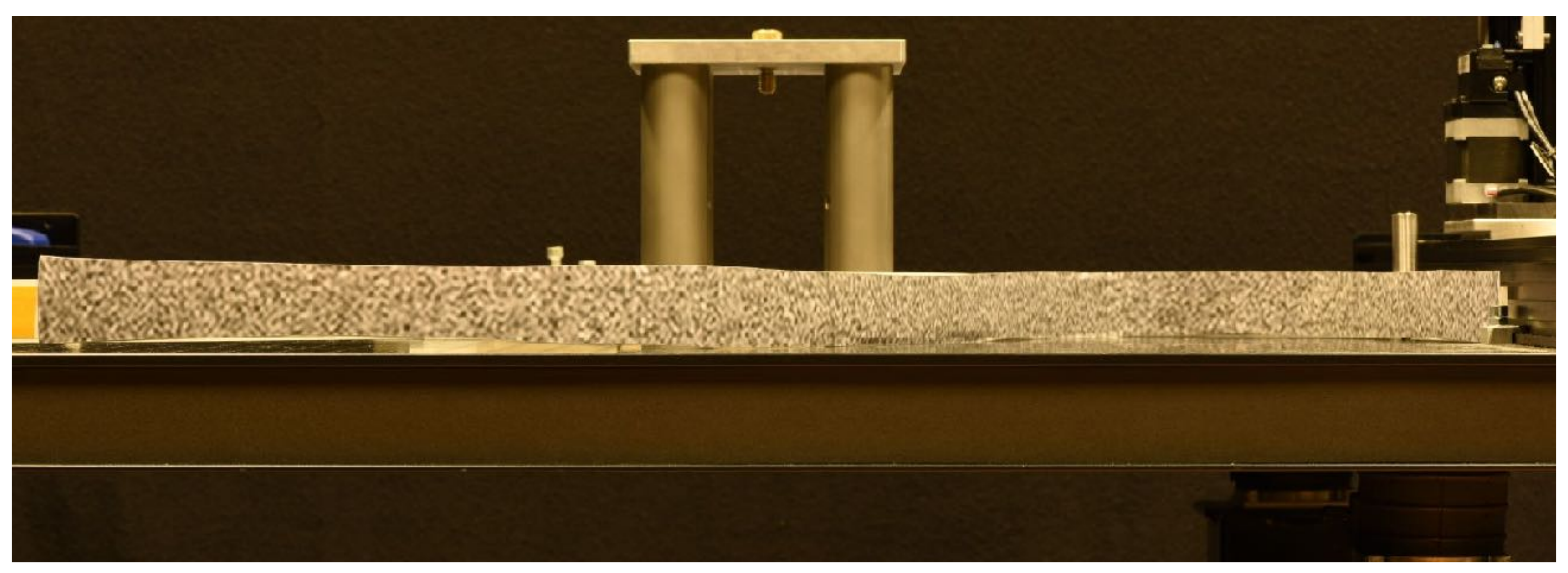

Intensity

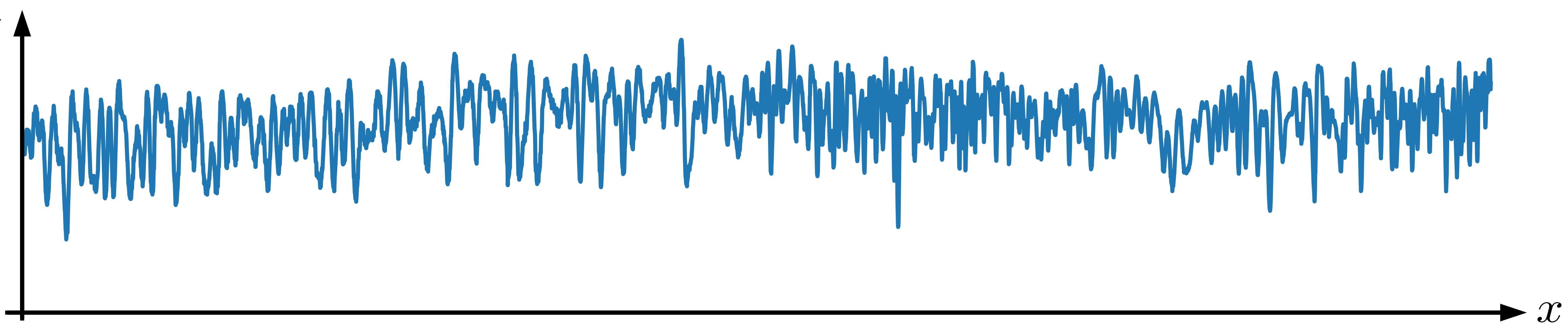




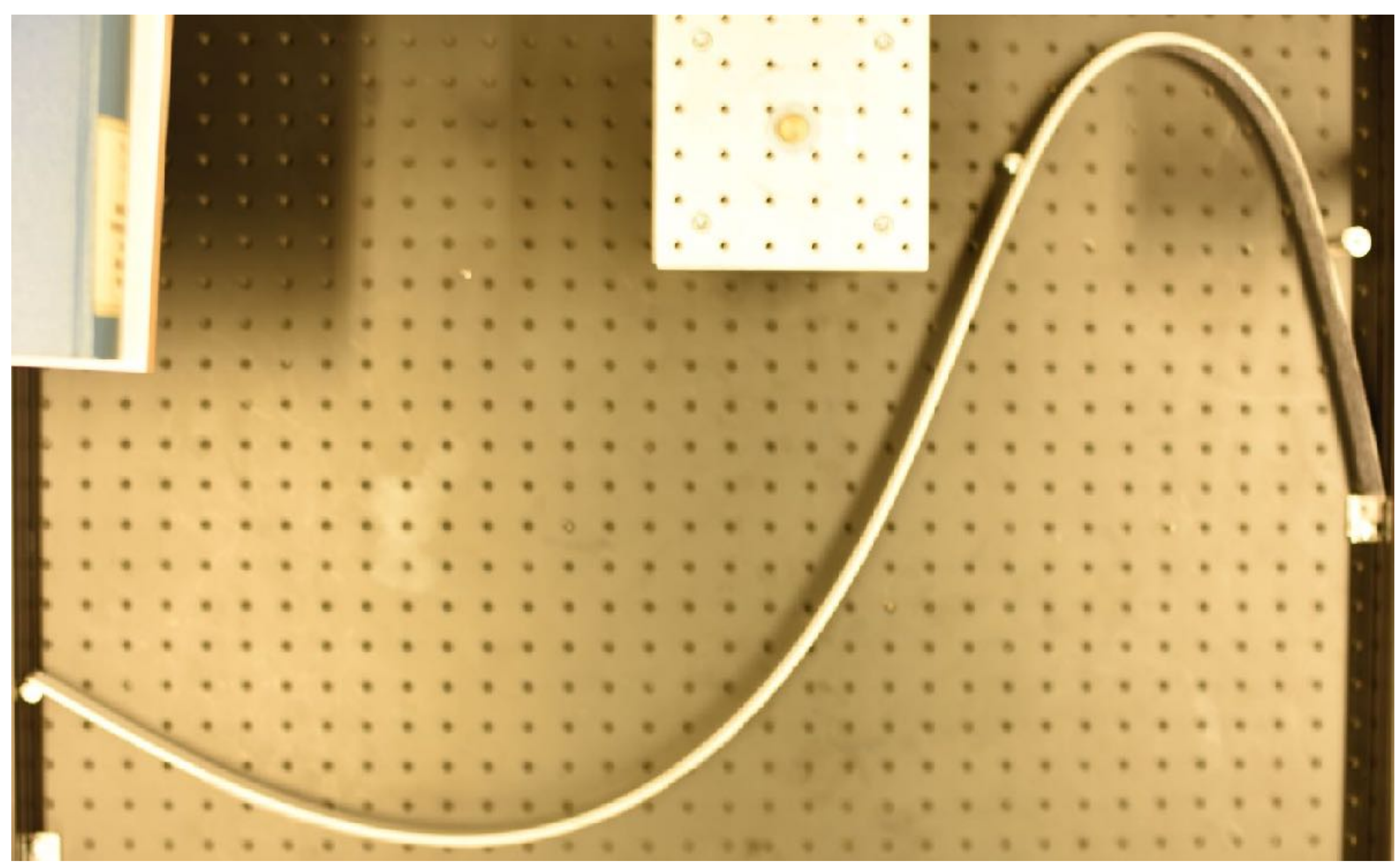




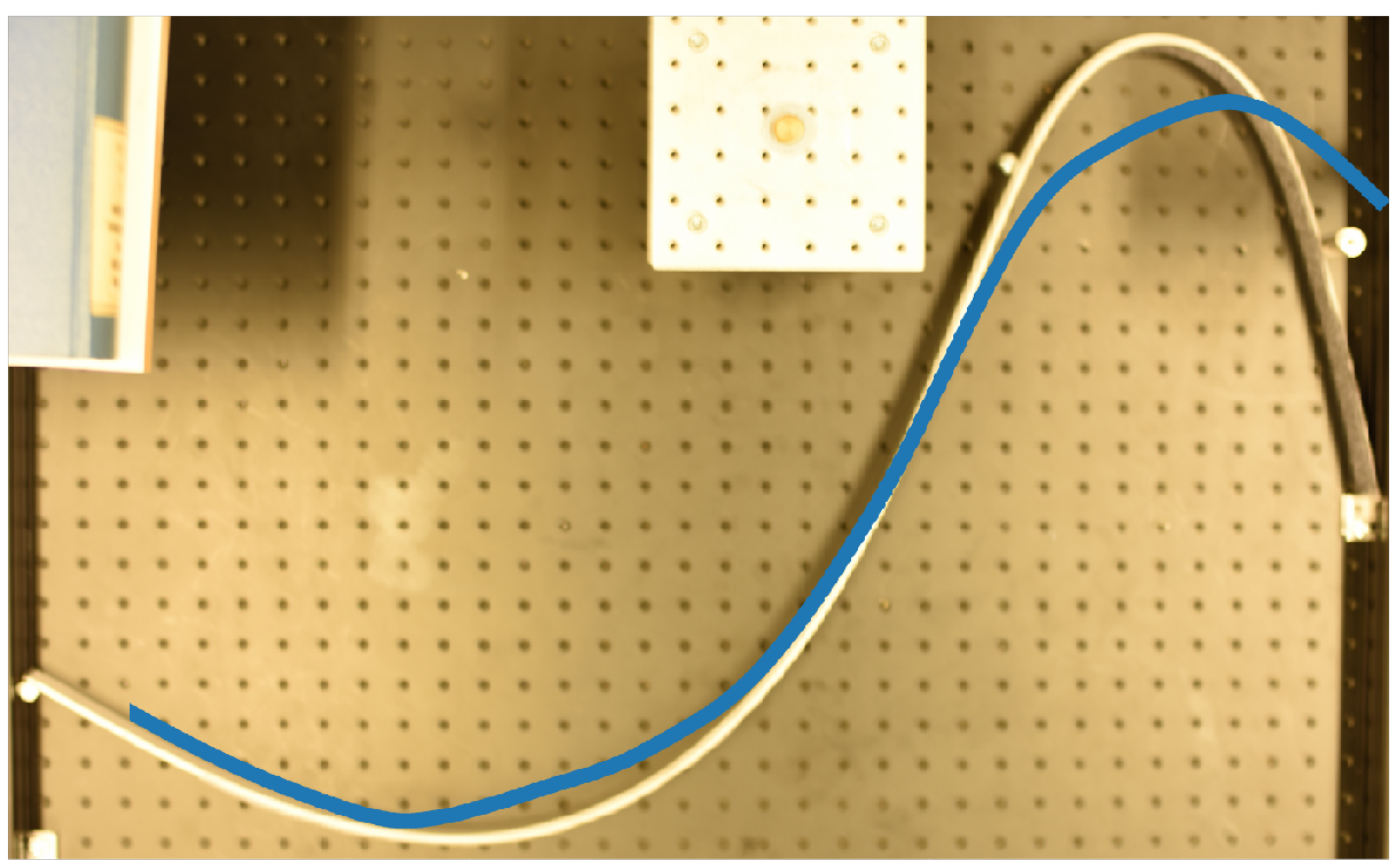


- Basic algorithm to retrieve surface from bandwidth

\section{Future work}

- Extensions - Central projection and 3D

- Algorithmic improvements - window size ...

- Sampling

- Uniqueness and recovery guarantees

- Related - calibration, structured light depth sensing ... 


\section{Thank you}

An IPython notebook is available reproducing all the results of the paper: https://infoscience.epfl.ch/record/224065?/n=en

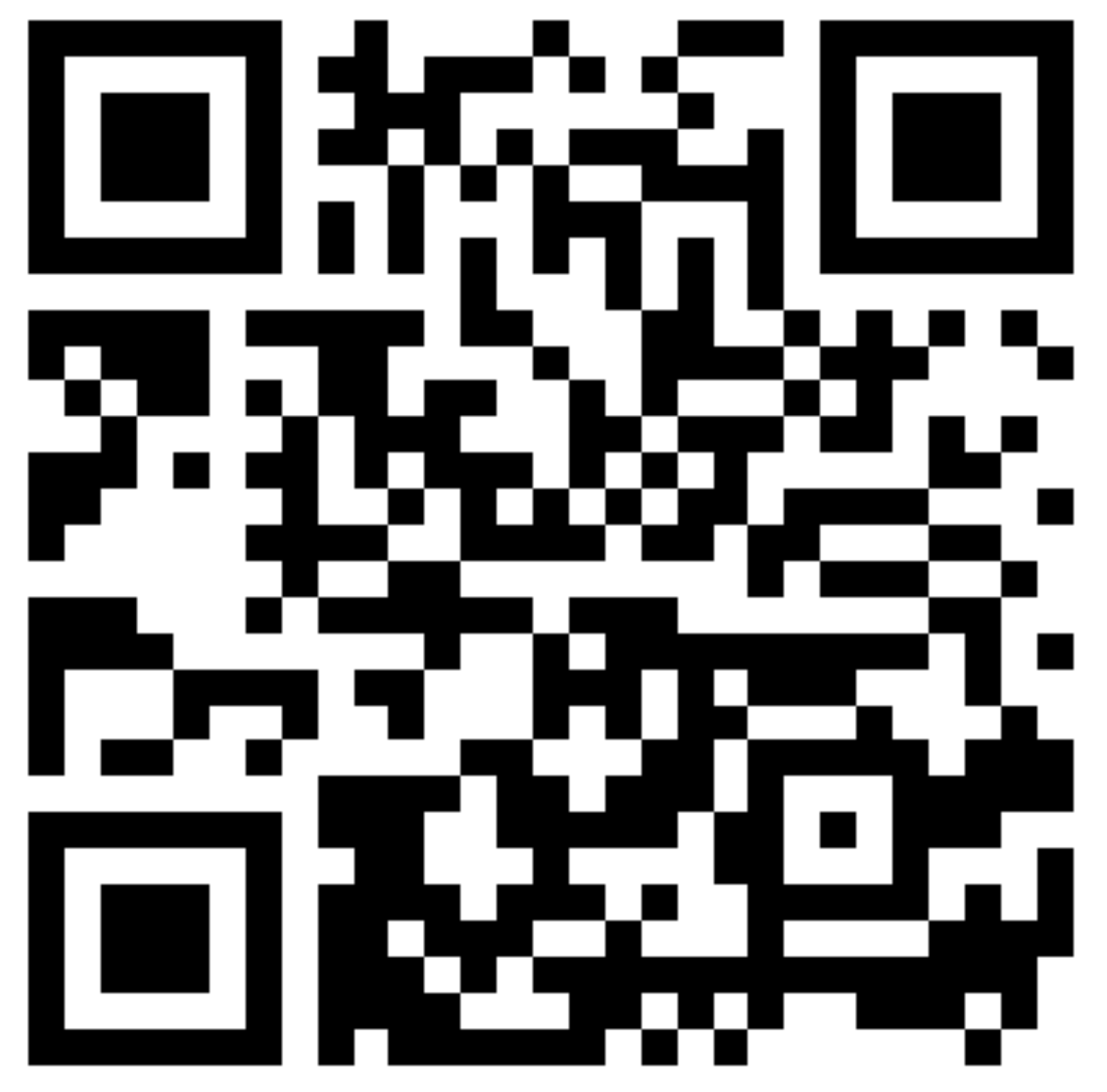


Sinusoidal texture 
Sinusoidal texture

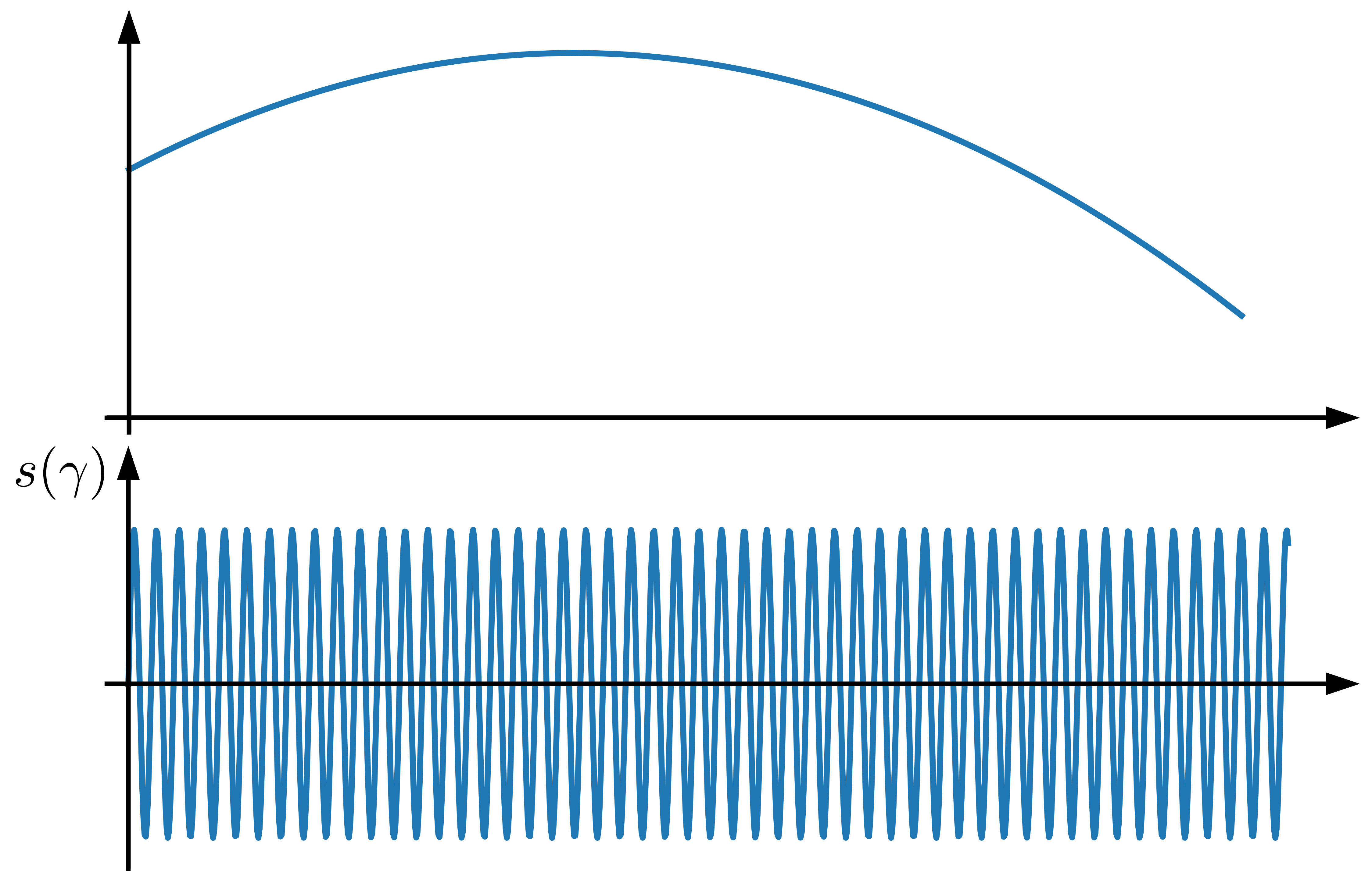


Sinusoidal texture

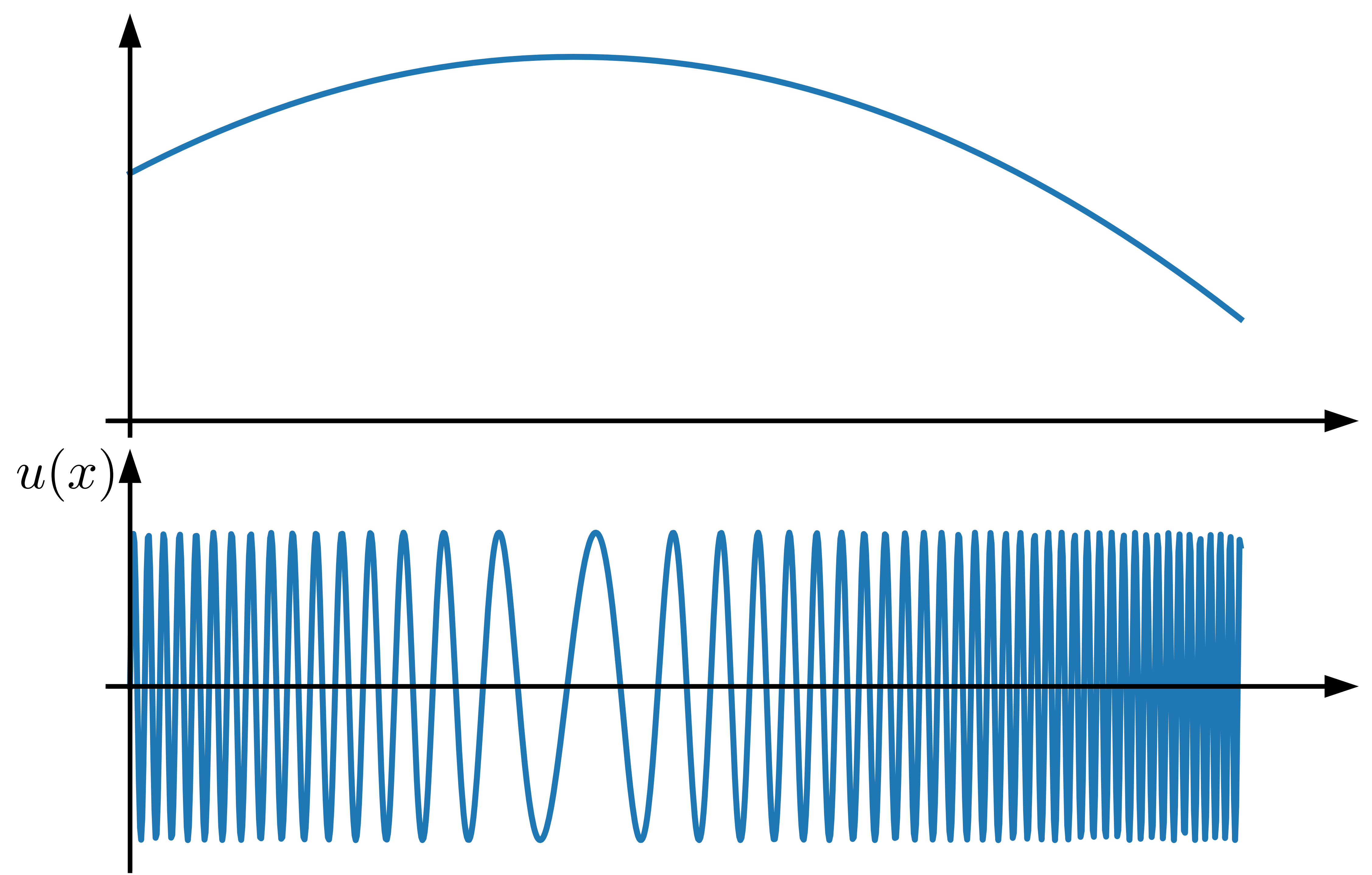




$$
\begin{gathered}
s(\gamma)=A \sin (\omega \gamma) \\
\frac{d u}{d x}=A \omega \frac{d \gamma}{d x} \cos (\omega \gamma(x))
\end{gathered}
$$

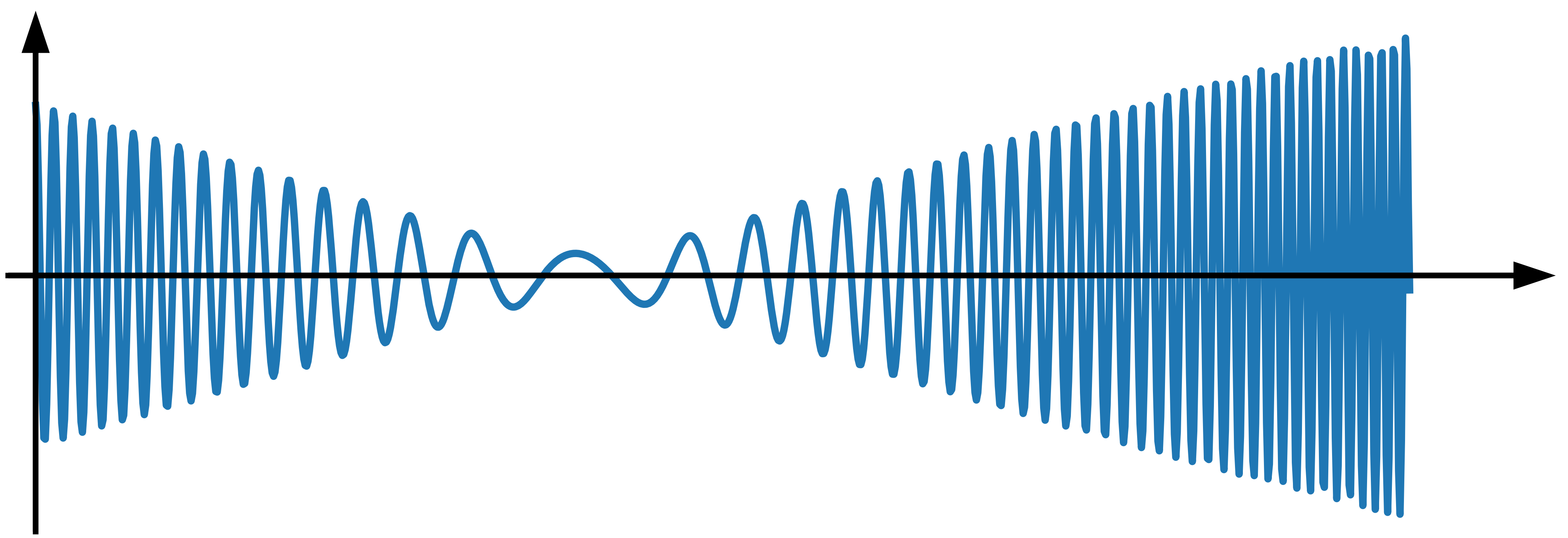




\section{Sinusoidal texture}

$$
\begin{gathered}
s(\gamma)=A \sin (\omega \gamma) \\
\frac{d u}{d x}=A \omega \frac{d \gamma}{d x} \cos (\omega \gamma(x))
\end{gathered}
$$

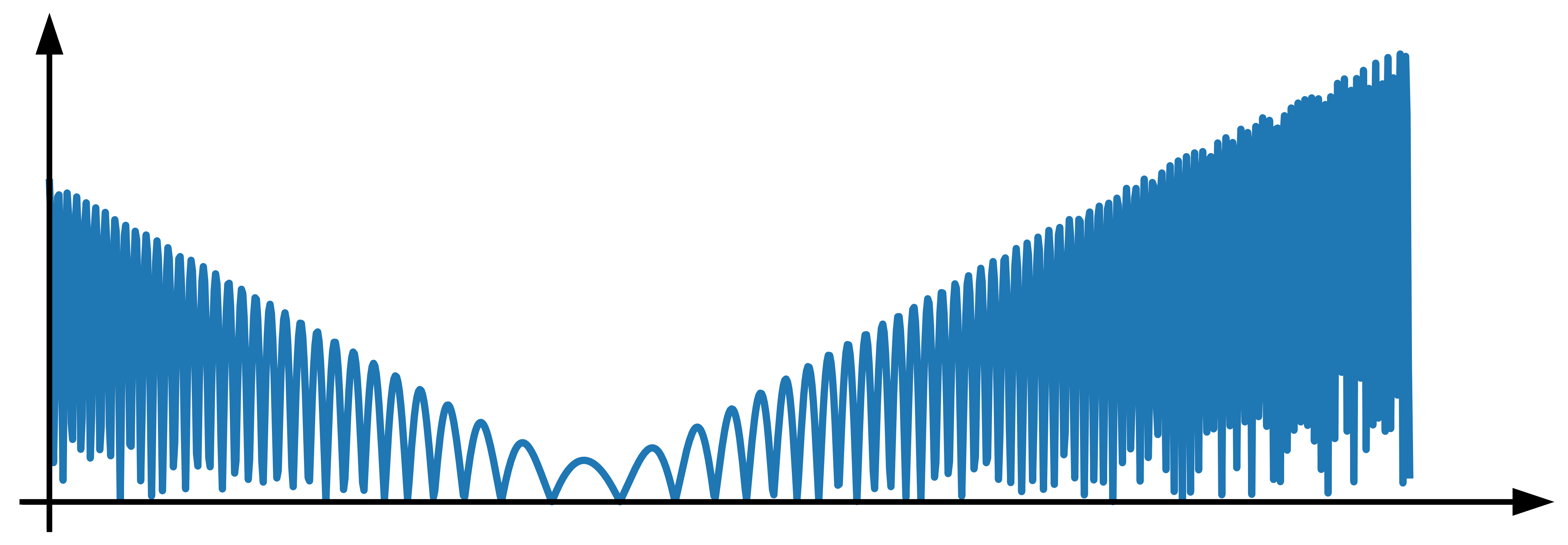




\section{Sinusoidal texture}

$$
\begin{gathered}
s(\gamma)=A \sin (\omega \gamma) \\
\frac{d u}{d x}=A \omega \frac{d \gamma}{d x} \cos (\omega \gamma(x))
\end{gathered}
$$

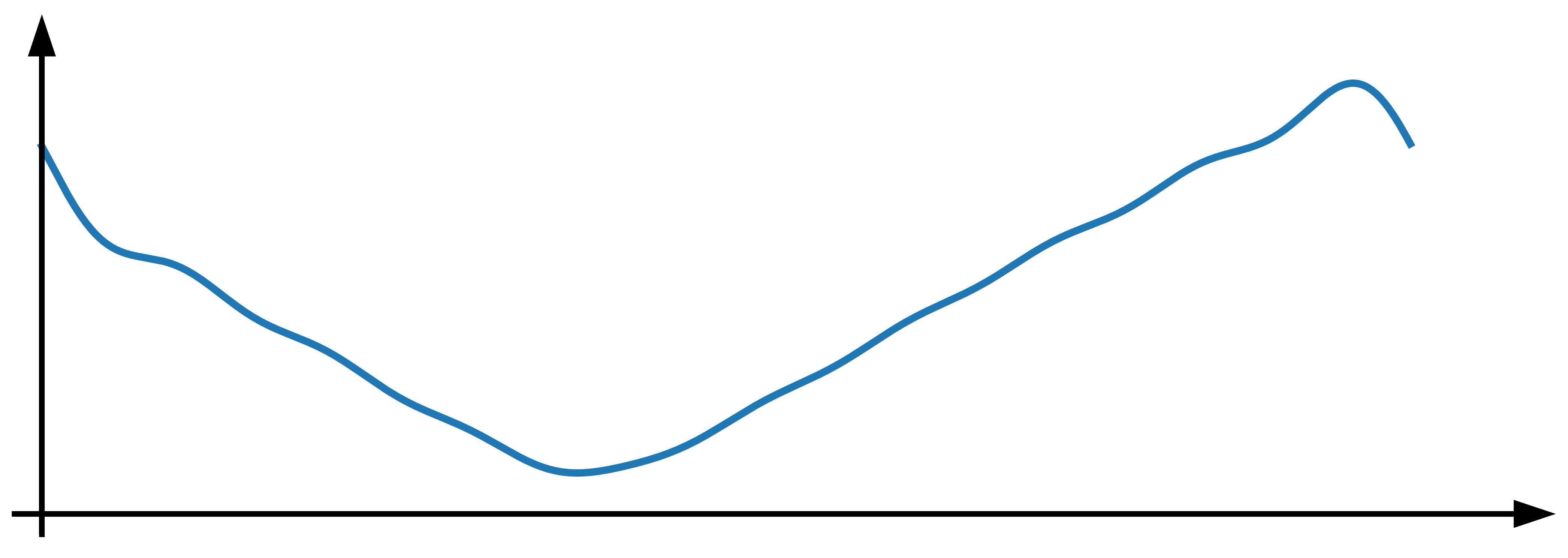




\section{Sinusoidal texture}

$$
\begin{gathered}
s(\gamma)=A \sin (\omega \gamma) \\
\frac{d u}{d x}=A \omega \frac{d \gamma}{d x} \cos (\omega \gamma(x))
\end{gathered}
$$

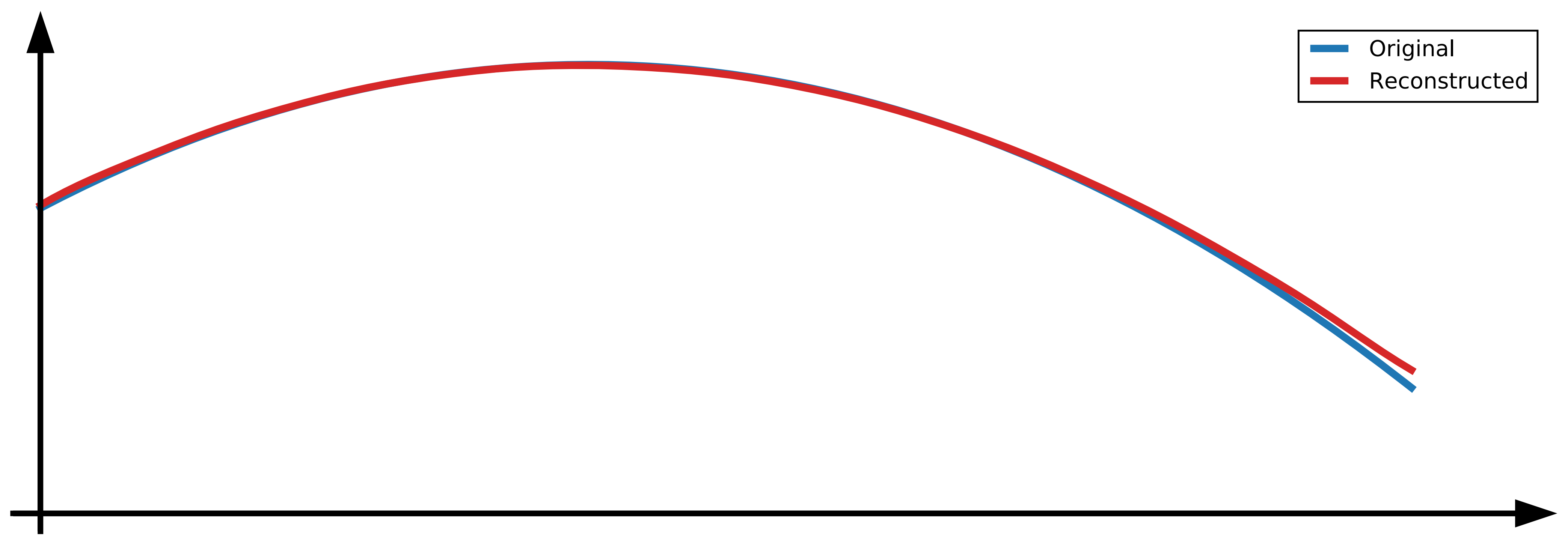

